\title{
Research on Siemens S7-200PLC Stacker Control Experiment Platform
} Jian Song ${ }^{1, a^{*}}$, Xiaojian Wang ${ }^{2, b}$, Fuxiang Xie ${ }^{1, c}$ and Qun Zhang ${ }^{3, d}$

1.College of Mechanical and Electrical Engineering, Weifang University, Weifang 266041, China

2. Electrical and Mechanical College, Weifang Vocational College, Weifang, 262737, China

3.Collage of Mechanical and Electronic Engineering, Shandong University of Science and Technology, Qingdao 266590, China

a524893101@qq.com, b1049535992@qq.com, c472934914@qq.com, d547711304@qq.com

Keywords: Stacker; PLC; Experimental platform; Institutional design; Electrical system

\begin{abstract}
According to the main development trends and characteristics of mechatronics and logistics specialty, aiming at the demand of experimental platform of stacker in automated warehouse, the performance analysis and design of stacker's experimental platform was studied and the experimental platform of stacker was determined overall objective, stacker mechanism, stacker control system hardware and software as well as safety protection device design and so on, carried on the stacker experimental platform control structure and the control method design. The experimental platform is reasonable in structure, concise and innovative, and can be widely used in experiment and innovative practice of electromechanical and logistics major in colleges and universities.
\end{abstract}

\section{Introduction}

With the continuous improvement of the degree of industrial automation and the rapid development of e-commerce platform, the stacker has been widely used in the automated warehouse in various industries. Stacker control is a mechatronic system that integrates mechanical, electronic, computer and automatic control technology. Due to its advantages of high space utilization efficiency, high efficiency of accessing goods, the use of computer for control management is more conducive to the realization of modern management of enterprise logistics, Has become an indispensable enterprise production logistics and transport equipment. [1.2] Based on the analysis and research of the stacker control system, this paper developed an experimental stacker automation platform which can be used in experimental training and innovative design in the field of electromechanical and logistics professionals to enable more students to In the experiment training, mastering the whole process of stacking and stacking machine in and out of the warehouse and the principle and method of the stacker control system, so that students can further handle the PLC's ability to control various practical problems of the stacker.

\section{Stacker Experimental Platform to Determine the Overall Objectives of the Program}

Nowadays, most of the colleges and universities in China use the small platform warehouse stacker experimental platform equipment, which is made by the professional production of teaching equipment manufacturers, which features a stacker using precise positioning of the stepper motor Servo motor as a driving force, with a small footprint, you can directly purchase the advantages of products at the scene, but the main problems exist high prices, experimental conditions and application conditions are a big gap. Considering the experimental results, equipment cost, occupied space, equipment operation and maintenance and other aspects of the actual situation of mechanical engineering training center, decided to independently developed by the stacker control experimental platform program. 


\section{Stacker Experimental Platform for the Technical Requirements and Functional Requirements}

Stacker experimental platform to ensure the strength, stiffness and reliability of the premise, as far as possible the use of lightweight materials to reduce the stacker weight and reduce unnecessary equipment to reduce the volume, making the stacker structure is simpler, more beautiful appearance, Run safer, more convenient operation. [3,4]

Stacker experimental platform main technical requirements are:

Speed requirements: Stacker horizontal motion speed up to $40 \mathrm{~m} / \mathrm{min}$; stacker horizontal motion speed up to $25 \mathrm{~m} / \mathrm{min}$; fork speed of $5 \mathrm{~m} / \mathrm{min}$.

Lift force requirements: no-load, to enhance the weight of $10 \mathrm{~kg}$ goods, to enhance the weight of $50 \mathrm{~kg}$.

The number of cargo requirements: a roadway; two rows of shelves; each row has 6 layers 7 columns, a single row has 42 cargo spaces, a total of 84 two rows of cargo space.

Stacker experimental platform to achieve the function:

Stackers to achieve three directions of movement, that is, stackers horizontal movement, the vertical movement of the cargo table, left and right forks access movement.

Stacker can be manual and automatic control of two ways, in the equipment debugging and emergency manual control, the normal access to the stacker automatic control of the goods.

Use the remote touch screen man-machine interface of a variety of virtual key switch to the S7-200PLC send a variety of operating instructions to control the stacker manual and automatic control, but also in the stacker control cabinet button switch off to send to the S7-200PLC A variety of control instructions to achieve reliable mechanical control. [5]

Stacker can automatically select the low, medium and high speed mode according to the actual running distance.

\section{Stacker Experimental Platform Design}

\section{Stacker Mechanism Design}

The structure of the stacker includes a rack, a horizontal movement and vertical lifting mechanism, a loading platform and a fork mechanism, etc. The rack structure of the stacker adopts a single column structure, the single column structure meets the requirements of the weight of the goods to be lifted, and Single-column stacker rack structure is more compact, operational field of vision, most of the stacker used in the form. Stacker structure shown in Figure 1.

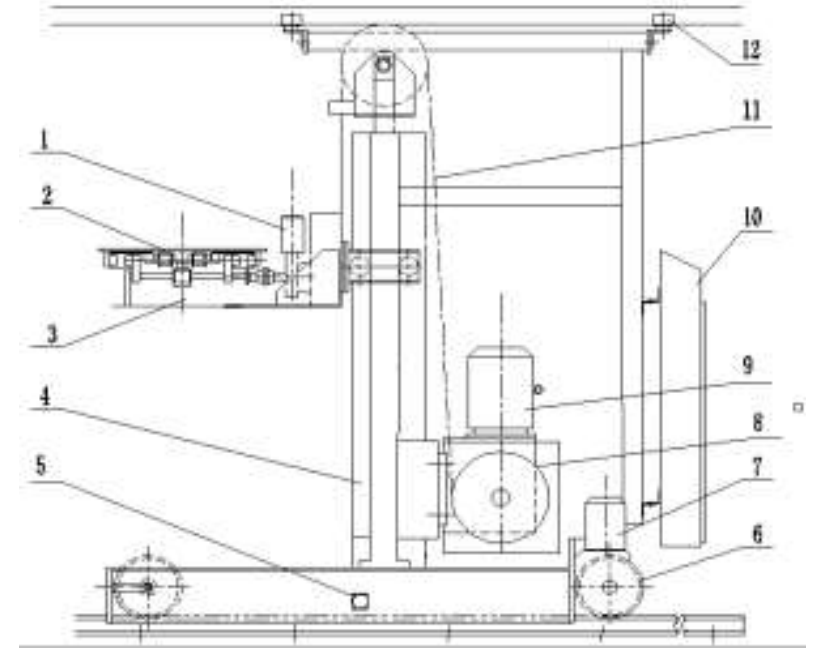

Figure 1 Stacker structure diagram

Figure 1 Notes: 1 table motor; 2 fork; 3 cargo table; 4 column; 5 laser range finder; 6 horizontal walking wheel; 7 three-phase asynchronous motor; 8 reducer; 9 servo motor; 10 control cabinet 11 Rope 12 Upper guide wheel 
The main function of the horizontal running mechanism is to realize the horizontal movement of the stacker along the guideway in the laneway. Most commonly used stacker running mechanisms have an upper walking type suspension and a shelf support type. The stacker designed in this paper adopts The lower end of the drive is supported by the upper end of the guide type walking mechanism, this structure allows the lower part of the load to withstand running, the upper end of the guide can also withstand part of the load, improve the stability and stability of the stacker. [6,7,8] The structure shown in Figure 1. Stacker horizontal movement through the gear drive to drive the active walking wheel to achieve, in the horizontal beam under the carriage installed three-phase asynchronous motor as a walking motor, in order to save axial space, the motor through the reducer according to a fixed transmission ratio through the gear Engage and then drive the driving wheel. The guide rails are in the form of upper rails and lower rails. The bottom of the stacker is equipped with a walking guide wheel at one end and a traveling drive wheel at the other end to ensure that the stacker runs along the lower rails.

The main function of the vertical lifting mechanism is to realize the vertical movement of the stacker's loading platform. The commonly used lifting mechanism of the stacker is towed by the flexible chain of the lifting chain. The lifting mechanism designed in this paper adopts the winding wheel to drive the steel wire rope Lifting scheme, the wire rope is led out by the winding wheel, and after the fixed pulley at the upper end of the pillar is changed to be directly connected with the loading platform, the loading platform is driven to move up and down along the column guide to drive the fork mechanism to ascend and descend, Taiwan arrived at the designated level accurately and smoothly parking, the structure shown in Figure 1.

The function of the forwarder is mainly to realize the access to the goods, which is the executive component of the stacker to access the goods. At present, the three-stage linear retractable fork mechanism is commonly used, which is composed of the upper, middle and lower trigeminal and the guiding support Rolling wheel bearing and so on. The mechanism can ensure that there is enough retractable stroke in the roadway so that the delivery and delivery of goods can reach the target shelf exactly, and the movement relation and structure are simple. In this paper, through the meshing transmission between gear and rack and the rope pulley The lower fork is fixed on the loading platform, the three-phase motor is used as the drive motor of the forks, the gear and the rack meshing transmission are used to convert the rotational movement of the motor into the rack through the reducer Of the linear motion, rack drive fork to achieve two-way telescopic movement. ${ }^{[4]}$ The fork mechanism is installed on the loading platform. The loading platform adopts the rolling guide wheel to clamp the column to limit the horizontal movement of the loading platform. The upright column is used as the guide rail for the vertical movement of the loading platform, The vertical direction of the column guide rail movements without the slightest horizontal shaking, the use of the guide roller platform guide than the sliding guide can greatly reduce the friction resistance, as shown in Figure 1.

\section{Stacker Electrical Control System Design.}

The electrical control system of the stacker includes hardware and software sections. Stacker control system hardware mainly Siemens S7-200PLC, Velen TK6070iH touch screen, Delta VFD-M-type inverter, Yaskawa SGDV servo controller and YASKAWA servo motor, laser range finder, photoelectric switch, signal detection Sensors and other components. The control cabinet is installed on the vertical column of the stacker, which effectively reduces the distance of transmitting the signal line feedback signal line and effectively improves the reliability of the system. The basic structure of the control system shown in Figure 2. The stacker control system software consists of the EB8000 project manager and STEP 7-MicroWIN / sp6 software. 


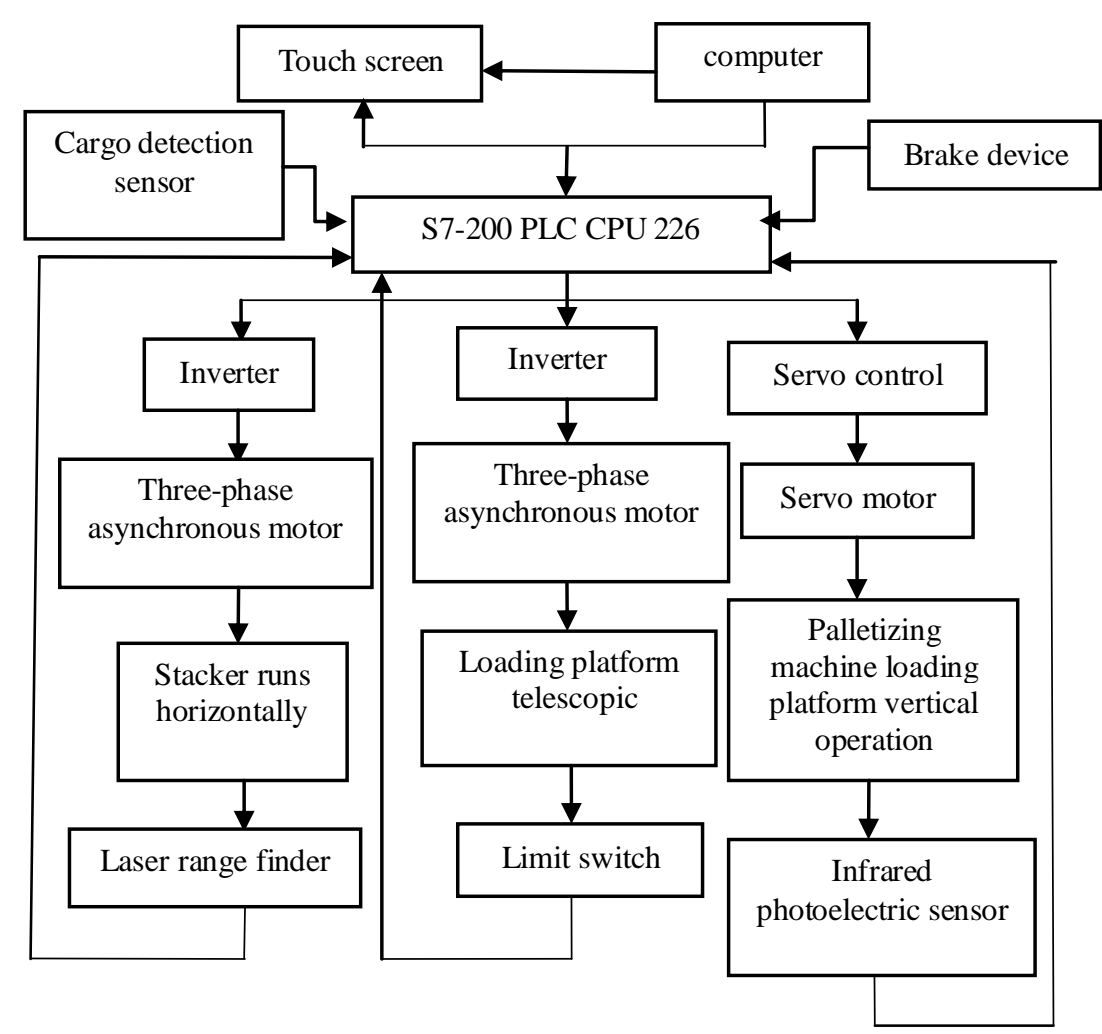

Figure 2 Basic structe of the control system

Stackers run by entering the corresponding access address on the touch screen, the address sent to the S7-200PLC through the RS485 communication line, PLC based on the current position of the stacker is detected, the signal sent to drive the stacker movement, Into the inverter and servo controller, drive motor movement, to achieve stacker movement. [7,8] The stacker uses two types of control, manual and automatic. Stacker control system software design deposit shown in Figure 3.

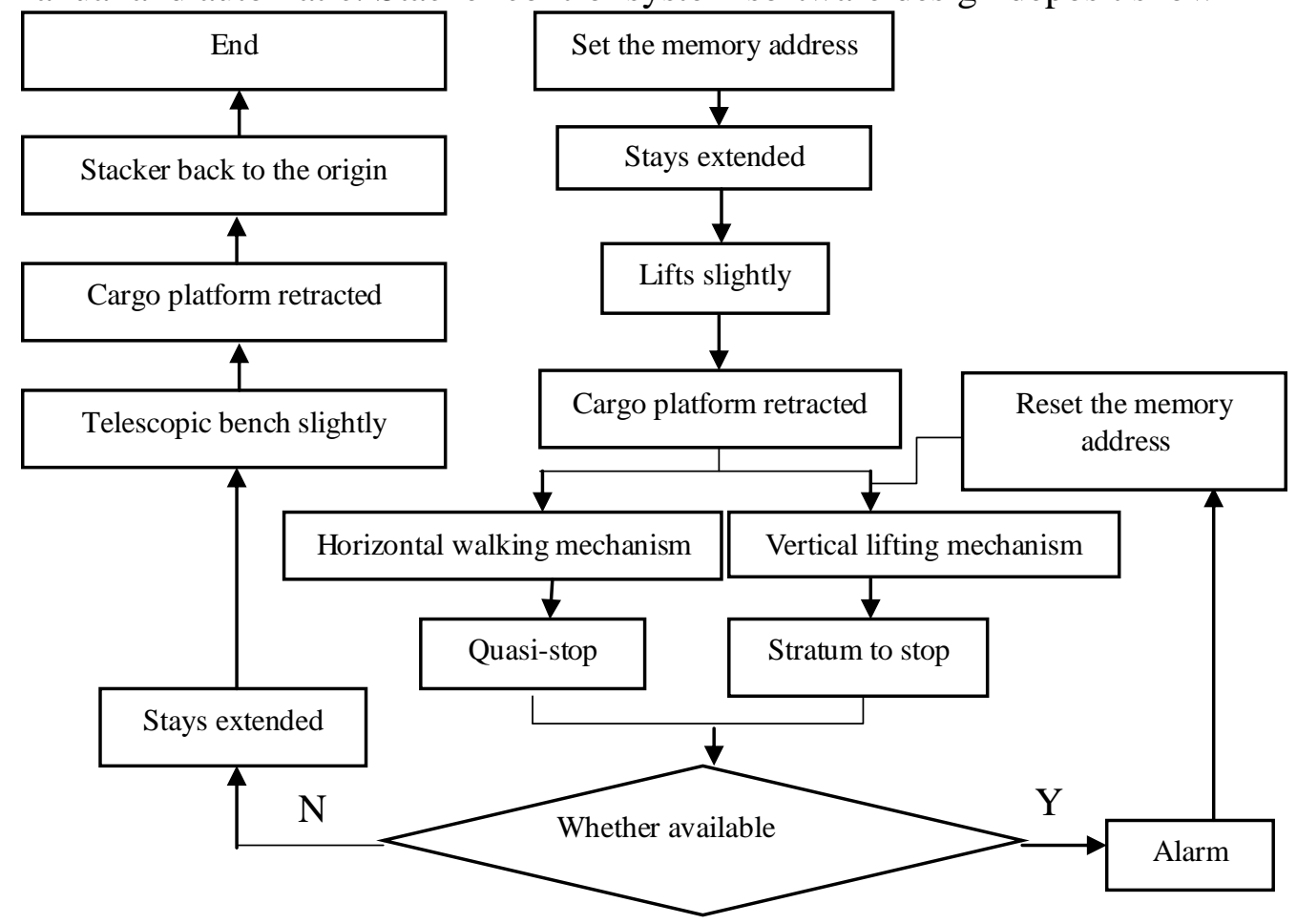

Figure 3 Stacker inventory flow chart 
Stacker inventory workflow: Enter the address number of the inventory to be stored on the touch screen, and send the serial number input to the port of S7-200PLC via RS485 communication line. The S7-200PLC sends the pulse signal to the inverter and servo controller. After the palletizer picks up at the origin, it moves to the target position. When the target position is detected, the palletizer stops moving and detects the presence or absence of the goods. In the absence of the goods, the forks reach the shelf stock, load Cargo platform slightly down, fork retracted, the goods saved to the target location, and then stacker back to the origin standby. This completes the inventory process.

Stacker pick up workflow: Enter the address number to pick up on the touch screen, the number entered by the RS485 communication line to the S7-200PLC port0, S7-200PLC to the drive and servo controller to send a pulse signal. Stacker in horizontal walking wheel driven by the completion of horizontal movement, the loading platform in the rope to complete the vertical movement under traction. After arriving at the designated address, the forks protrude, the loading platforms slightly increase and the forks retract. After picking up, the S7-200PLC drives the stacker back to the origin. After reaching the origin, the fork is extended, the loading platform is slightly lowered, the fork is retracted, and the goods are placed at the pickup platform. The stacking machine is on standby. To complete the pick up process.

\section{Stacker Control Experiment Platform Features}

Self-developed stacker control experiment platform with independent intellectual property rights, the technology and knowledge involved can be demonstrated in all directions in front of teachers and students, stacker control platform involves mechanical, electronic, computer technology, automatic control technology and control system Design and other aspects of knowledge. The experimental platform can be applied to the field of electromechanical control and logistics control related fields, to facilitate students to comprehensively practice the comprehensive ability to expand. Combining with the specific environment of training center, the functional requirements and performance requirements of the stacker experiment platform are well met, saving purchase cost to a great extent and improving the independent innovation ability of teachers and students.

\section{Conclusion}

This paper discusses the design scheme of stacker experiment platform, which largely makes up for the shortage of the combination of electromechanical and logistics specialty, integrates the major functions of mechanical, electronic, automatic control and logistics related major, With the least investment, completed the development of experimental platform. The test platform has a reasonable design, beautiful structure, high reliability and accuracy, as far as possible in the control system concise, so that sports performance is fast and efficient, and has practical value, is ideal for colleges and universities as a learning platform use.

\section{Acknowledgements}

This work is supported by Project of National Natural Science Foundation (No.51505337) and A Project of Shandong Province Higher Educational Science and Technology Program (No. J17KA150)。

\section{References}

[1] Y. Li: Study of Automatic Warehouse and Its Stacker(MS., Harerbin University of Science and Technology,China,2012),p.32-45.

[2] Q.S. Guo: Research on Mechanical Structure and Control System Design for Stacker(MS., Shenyang ligong University,China,2013),p.51.

[3] M.X. Gu, J.H. Bao, Y. Zhang, X. Jiang: Industrial Control Computer, Vol. 23 (2010) No.4, p.83 
[4] G.T. Ha: Electronics World, (2013) No.2, p.121.

[5] J.T. Wang, H.Q.Liu: Mechanical Engineering \& Automation, (2011) No.3, p.129.

[6] Germany's Siemens Company. S7-200 System Manual[K]. 2004

[7] Y.C. Zhao: Logistics Engineering and Management, Vol.31 (2009) No.1, p.40.

[8] S.L. Yao: Manufacturing Informatization, Vol.3 (2010) No.12, p.64. 